\title{
Actualización: Obesidad y dietas: algunas controversias
}

\author{
Obesity and diets: some controversies
}

Agustina Liaudat*

\begin{abstract}
Resumen
El estudio DIRECT'1 resumido y comentado en este número de EVIDENCIA, analizó el efecto de las dietas hipograsa, mediterránea y baja en hidratos de carbono o cetogénica, concluyendo que estas dos últimas alternativas podrían ser efectivas para la reducción de peso, en comparación a las dietas hipograsas ${ }^{\$ 2,3,4}$. A propósito de dicho trabajo, el presente artículo revisa aspectos epidemiológicos de la obesidad y los estilos de vida en Argentina, sus explicaciones etiopatogénicas actuales, algunos lineamientos generales de las principales estrategias de abordaje y las principales controversias respecto de las dietas cetogénicas.
\end{abstract}

\section{Abstract}

The DIRECT study, summarized and reviewed in this issue of EVIDENCIA, analyzed the effects of low-fat, Mediterranean, or low carbohydrate ( cetogenic) diets, concluding that the last two alternatives could be effective for weight reduction, as compared to low-fat diets. With this work in mind, the present paper reviews epidemiological aspects of obesity and lifestyle in Argentina, their current etiopathogenic explanations, some general guidelines regarding the main therapeutic strategies, as well as the main controversies about the cetogenic diets.

Palabras clave: obesidad, dietas, riesgo cardiovascular, dieta cetogénica. Key words: obesity, diets, cardiovascular risk, cetogenic diet.

Liaudat A. Obesidad y dietas: algunas controversias. Evid. actual. práct. ambul; 11(5): 147-148, Sep-oct.2008.

\section{Epidemiología de la obesidad y de los estilos de vida en Argentina}

La obesidad es una enfermedad crónica de difícil manejo consi-derada actualmente un problema de salud pública por su alarmante prevalencia, por sus consecuencias sobre la morbimortalidad y la calidad de vida, y por el elevado costo social y económico que supone. En Argentina, el Ministerio de Salud realizó la Encuesta Nacional de Factores de Riesgo ${ }^{5}$ (ENFR) y la Encuesta Nacional de Nutrición y Salud (ENNyS). Estos relevamientos informaron que más de la mitad de los hombres y mujeres mayores de 18 años están excedidos de peso; y que el $50 \%$ de los niños y niñas tiene sobrepeso, en coexistencia con desnutrición crónica o talla baja, y carencia de micronutrientes como zinc, calcio, hierro, vitamina $\mathrm{C}$ y $\mathrm{A}$. Con respecto a la práctica de ejercicio, el $46,2 \%$ de la población refirió realizar un nivel bajo de actividad física.

Un análisis publicado recientemente indica que en Argentina el consumo calórico medio se encuentra entre 2700 y $3100 \mathrm{kcal}$ por día y hace referencia a la preferencia de los consumidores por los alimentos obesogénicos, al posible impacto de una disminución en los precios de los productos saludables; y al escenario de posibles intervenciones del Estado ${ }^{6}$ vinculadas a la provisión de información nutricional adecuada, a la regulación de la publicidad, y a planes globales preventivos ${ }^{7}$.

La Ley de Obesidad fue aprobada en la Argentina en agosto de 2008. Además de incluir la cobertura de la obesidad en el Programa Médico Obligatorio (PMO, paquete de prestaciones obligatorio para el sistema de la seguridad social) la norma sancionada también establece directivas para combatir esta patología. Quizás sea este un primer paso hacia la implementación de políticas sanitarias de prevención global de esta epidemia.

\section{Comprendiendo la obesidad}

Distintos factores etiopatogénicos se relacionan con el sobrepeso y la obesidad: factores genéticos, anormalidades en el gasto energético y en la oxidación de grasas, regulación neuroendócrina, sobrealimentación, disminución en la actividad física y la acción de ciertos medicamentos. Actualmente van adquiriendo mayor relevancia el papel de los factores ambientales y los estilos de vida; haciendo todo este conjunto de factores que las medidas preventivas y terapéuticas no sean simples ni fáciles de aplicar.

\section{Estrategias para el abordaje}

La valoración de los pacientes con obesidad no debe realizarse solamente sobre la base de su sobrepeso sino también respecto de los riesgos asociados a dicha entidad clínica. Son de utilidad en este sentido, la medición de la circunferencia de la cintura, tener en cuenta la presencia de comorbilidades como diabetes, hipertensión arterial, dislipemia, síndrome metabólico y de otras condiciones clínicas que puedan alterar el desarrollo ponderal, influir en el riesgo cardiovascular global o dificultar las estrategias terapéuticas (ej. osteoartritis empeorada por un exceso de peso que, a su vez, dificulta la práctica de actividad física). La primera estrategia en el tratamiento de la enfermedad cardiovascular, la obesidad, la diabetes, la hipertensión y las dislipemias, es el plan alimentario y la modificación del estilo de vida $^{8,9,16}$. En este sentido, la indicación de pérdida de peso debe ser específica, realista y con un enfoque interdisciplinario. Además de la prescripción de dietas y de actividad física, contamos hoy en día con estrategias para motivar a los individuos a modificar sus hábitos de vida, así como con fármacos"10 y técnicas quirúrgicas para el tratamiento de la obesidad. La aplicación de las mismas requiere un esfuerzo considerable por parte del paciente, por lo que es esencial que esté motivado y apoyado por un equipo terapéutico, que se realice una adaptación dietética individualizada, recomendaciones personalizadas de actividad física, un asesoramiento conductual que le permita integrar los cambios establecidos en su entorno familiar y psicosocial; así como tratamientos adicionales, dependiendo del grado de sobrepeso y presencia de comorbilidades.

A lo largo del tiempo de su implementación, la mayoría de las dietas extremas generan grandes dificultades en la adherencia ${ }^{11}$. Por otro lado, la distribución ideal de macronutrientes para lograr un descenso de peso continúa siendo controvertida ${ }^{12}$

En este sentido, la dieta "cetogénica" se ha convertido en una opción de popular elección y surge como alternativa a los planes recomendados hasta el momento. Si bien esta dieta se fundamenta en que el plan hiperproteico y la cetosis generarían un efecto de saciedad que ayudaría a disminuir el ingreso energético; quedan por evaluar sus efectos en el largo plazo derivados de la cetosis, de los efectos secundarios al consumo elevado de grasas saturadas, del perfil lipídico resultante; así como su impacto en la actividad física, la hipercalciuria y el daño renal ${ }^{13}$ y el efecto en el riesgo de cáncer derivado de las opciones y omisiones alimenta- 
rias sugeridas en este tipo de programa alimentario ${ }^{14}$.

En el estudio DIRECT ${ }^{1}$, que resumimos y comentamos en este número de EVIDENCIA, se hace referencia a resultados saludables con descensos moderados en el peso, similares a los obtenidos con farmacoterapia. En este sentido vale aclarar que no es lo mismo la implementación de una dieta cetogénica a través de la inclusión de grasas de origen vegetal (ej. aceitunas, aceite de oliva, frutas secas, etc.) como pudo haber ocurrido en la población estudiada en Israel, que hacerlo sobre la base de la inclusión de alimentos de origen animal (ej. fiambres), lo que podría terminar resultando una tentación para los pacientes de nuestro medio, por cuestiones culturales, de disponibilidad y económicas. Por otro lado, la dieta cetogénica no parecería ser más costo-efectiva que la dieta hipocalórica estándar ${ }^{15}$

Actualmente, algunas dietas cetogénicas también promocionan la incorporación de fibras, hidratos de carbono de bajo índice glucémico y mejores opciones en la calidad de las grasas. Pero continuamos sin saber los efectos de la eliminación de grupos de alimentos a largo plazo. Y dentro de este nuevo enfoque quizás termine acercándose la dieta cetogénica a las recomendaciones de una dieta mediterránea.

Conseguir un peso ideal resulta en la mayoría de los pacientes un objetivo inalcanzable. Los cambios frecuentes de peso -vulgarmente conocidos como "efecto yo-yo"- y dietas muy restrictivas pueden precipitar trastornos alimentarios, respuestas fisiológicas que limitan la pérdida de peso y fracasos repetidos. Se ha demostrado que las pérdidas de peso moderadas -un 5 a $10 \%$ por debajo del peso inicial- se asocian a una mejoría de las comorbilidades y a una disminución de la mortalidad ${ }^{16,17,18,19}$. Por ejemplo, una disminución inicial de 500 a $1000 \mathrm{kcal}$ diarias logra un descenso de peso aproximado de 250 a 500 gramos por semana durante seis meses. En este sentido, podemos resumir que el objetivo principal es mejorar la salud general del individuo y no sólo su peso, haciendo hincapié en el monitoreo continuo del paciente ${ }^{17}$

Las recomendaciones generales en las guías de tratamiento de la obesidad y de prevención de la enfermedad cardiovascular tien- den a un descenso de peso y de la circunferencia de cintura, a través de cambios en el estilo de vida, de una dieta hipocalórica con un 45 a $55 \%$ de hidratos de carbono, 15 a $25 \%$ de proteínas, 25 a $35 \%$ de grasas -con menos de $7 \%$ saturadas, 15 a $20 \%$ monoinsaturadas, menos de $7 \%$ poliinsaturadas y menos de $2 \%$ de grasas trans- y 20 a 40 gramos diarios de fibra ${ }^{20,21,22}$.

Vale destacar las conclusiones de un importante estudio longitudinal llevado a cabo en Europa (El proyecto HALE) el cual concluyó que la adhesión a una dieta mediterránea y a un estilo de vida saludable se vio asociada a más de un $50 \%$ de disminución de la mortalidad por todas las causas ${ }^{23}$. Es importante señalar de todos modos que estudios observacionales como el HALE pueden sufrir de importantes sesgos, y no reemplazan la evidencia proveniente de ensayos clínicos aleatorizados.

\section{Conclusiones}

El éxito del tratamiento no debe solo valorarse en función del peso sino también en las mejoras de la comorbilidad, en la calidad de vida y en la integración social.

$\mathrm{Si}$ bien el plan alimentario es esencial en la prevención y tratamiento de la obesidad en cualquiera de sus etapas; los predictores de mantenimiento incluyen el automonitoreo del peso y el registro alimentario, la práctica regular de actividad física vigorosa y la restricción calórica, junto con el control del equipo terapéuti$\mathrm{CO}^{14}$. Todas las dietas requieren supervisión nutricional y es difícil mantenerlas día a día sin planificación. Si la dieta no se adapta al estilo de vida del paciente y a su contexto cultural, terminará siendo abandonada.

Respecto de las "dietas cetogénicas" es necesario contar con estudios controlados, con poblaciones más numerosas y con un seguimiento a más largo plazo para que las asociaciones científicas se decidan a avalarla. Sin embargo, por el momento, su uso durante períodos cortos y en pacientes con dificultades para el logro de objetivos parecería no presentar efectos adversos de importancia.

Recibido el 02/03/07 y aceptado el 18/11/07

Referencia

1. Shai I y col. Weight Loss with a Low-Carbohydrate, Mediterranean, or Low-Fat Diet., for the Dietary Intervention Randomized Controlled Trial (DIRECT) Group. N Engl J Med July 17, 2008; Volume 359:229-241.

2. Foster $\mathrm{G}$ y col. A randomized trial of a low-carbohydrate diet for obesity. N Engl J Med 2003;348:2082-2090.

3. Nordmann A, y col. Effects of low-carbohydrate vs low-fat diets on weight loss and cardiovascular risk factors: a meta-analysis of randomized controlled trials. Arch Intern Med 2006;166:285-293. [Erratum, Arch Intern Med 2006;166:932.]

4. Gardner $\mathrm{C}$ y col. Comparison of the Atkins, Zone, Ornish, and LEARN diets for change in weight and related risk factors among overweight premenopausal women: the A TO Z Weight Loss Study: a randomized trial. JAMA 2007;297:969-977. [Erratum, JAMA 2007;298:178.]

5. Encuesta Nacional de Factores de Riesgo. Ministerio de Salud de la Nación. 2005. Principales resultados resumidos en: Rubinstein A. Factores de riesgo y beneficios económicos del mejoramiento de la salud. Evid. actual. práct. ambul; 10(4): 98-100, jul-agos.2007. Disponible en URL: http://www.foroaps.org/files/edit\%2010\%204.pdf (último acceso 19/09/08).

6. Rubinstein A, García Martí S. y col. Evaluación Económica Sectorial de un paquete de intervenciones preventivas para reducir la morbimortalidad cardiovascular en la ciudad

de Buenos Aires. Instituto de Efectividad Clínica y Sanitaria (IECS) y Programa VIGI+A. Ministerio de Salud de la Nación. 2005

Resumido y citado por: Rubisntein A. Definición de prioridades para la implementación de intervenciones sanitarias: enfermedades transmisibles (última entrega). Evid. actual. práct. ambul; 10(6):162-165, Nov-dic.2007. Disponible en URL: http://www.foroaps.org/files/fyufouy.pdf (ultimo acceso 19/09/08).

7. Britos S. Obesidad en la Argentina, también una cuestión económica. Disponible en URL: http://www.nutrinfo.com/pagina/info/obesidad_argentina.pdf (último acceso 19/08/08).

8. Assessment and manangement of adult obesity- AMA 2007. Citar en forma completa.

9. The JNC 7 Report. JAMA 2003; 289:2560-2571.

10. Rucker D y col. Long term pharmacotherapy for obesity and overweight: updated meta-analysis BMJ 2007;335:1194-1199.

11. Dansinger M y col. Comparison of the Atkins, Ornish, Weight Watchers, and Zone Diets for Weight Loss and Heart Disease Risk Reduction: A Randomized Trial. JAMA. 2005;293:43-53.

12. Finer N. Low-Calorie Diets and Sustained Weight Loss. Obesity Research 9: 290S-294S; doi:10.1038/oby.2001.133.

13. Reddy S y col. Effect of low-carbohydrate high-protein diets on acid-base balance, stone-forming propensity, and calcium metabolism. AJ Kid Diseases 2002; 40:265-274.

14. American Institute for Cancer Research. World Cancer Research Fund. Food, Nutrition, Pysical Activity and the Prevention of Cancer: a Global Perspective. 2007. Disponible en URL: http://www.dietandcancerreport.org/?p=ER (último acceso 19/09/08).

15. Gilden A y col. Cost-Effectiveness of a Low-Carbohydrate Diet and a Standard Diet in Severe Obesity. 120 Obesity Research (2005) 13, 1834-1840; doi: 10.1038/oby.2005.223.

16. Poirier G. Obesity and Cardiovascular Disease: phatophysiology, evaluation and effect of weight loss: an update of de 1997 American Heart Association scientific Statement on obe-

sity and heart disease from the Obesity Committee of the Council of Nutrition, Physical Activity and Metabolism. Circulation 2006; 113:898-918.

17. Wing P. Long term weight loss mantenance. Am J Clin Nutr 82; 222S-225S.

18. Eckel R. Nonsurgical Management of Obesity in Adults. N England J Med 2008; 358: 1941-1950.

19. Consenso SEEDO 2007 para la evaluación de sobrepeso y obesidad y el establecimiento de criterios de intervención terapéutica. Rev Esp Obes 2007; 5(3): 135-175.

20. Third Report of the Expert Panel on Detection, Evaluation, and Treatment of High Blood Cholesterol in Adults (ATP III Final Report). 2004. NHLBI

21. The DASH Plan. NHLBI. Revised 2006. Disponible en URL una versión para la comunidad: http://www.nhlbi.nih.gov/health/public/heart/hbp/dash/new_dash.pdf (último acceso $19 / 09 / 08)$

22. Evidence Based Guidelines for Cardiovascular Disease Prevention in Women AHA Guidelines. Circulation 2004; 109: 672-693.

23. Knoops K y col. Mediterranea Diet, Lifestyle Factors and 10-Year Mortality in Elderly European Men and Women. The HALE Proyect. JAMA 2004; 292: 1433-1439.

** Por ejemplo, un meta-análisis que incluyó estudios de uno a cuatro años de duración, orlistat, sibutramina y rimonabant se asociaron a una reducción moderada del peso (menos de $5 \mathrm{~kg}$ ) con diferentes efectos en los factores de riesgo cardiovascular y en los posibles efectos adversos. Sin embargo, la tasa de abandono de los tratamiento es alta y todavía no hay datos sobre los efectos en el largo plazo sobre la morbilidad y la mortalidad. 